\title{
Extracellular volume fraction in dilated cardiomyopathy patients without obvious late gadolinium enhancement: comparison with healthy control subjects
}

\author{
Dong Jin $\mathrm{Im}^{1^{*}}$, Yoo Jin Hong ${ }^{1}$, Chul Hwan Park ${ }^{2}$, Byoung Wook Choi ${ }^{1}$, Tae Hoon Kim²
}

From 18th Annual SCMR Scientific Sessions

Nice, France. 4-7 February 2015

\section{Background}

To evaluate whether the extra-cellular volume fraction (ECV) measured using cardiac MRI can detect myocardial tissue changes in dilated cardiomyopathy (DCM) without late gadolinium enhancement (LGE).

\section{Methods}

Forty-one DCM patients and 10 healthy volunteers underwent pre- and post- T1 mapping using a modified LookLocker inversion recovery (MOLLI) sequence, LGE, and cine MRI on a 3-T cardiac magnetic resonance (CMR) system. LGE-MR findings were used to divide DCM patients into two groups: Group A had no apparent LGE, and Group B had LGE apparent in at least one segment. The ECV of left ventricle (LV) myocardium (16 segments) was calculated in short-axis view as follows: $\mathrm{ECV}=[(\Delta \mathrm{R} 1$ of myocardium/ $\Delta \mathrm{R} 1$ of LV blood pool)] $\times$ (1 - hematocrit), where $\mathrm{R} 1=1 / \mathrm{T} 1, \Delta \mathrm{R} 1$ = Post-contrast $\mathrm{R} 1$ - Pre-contrast R1. The LV ejection fraction (LVEF) was obtained from cine MRI images. The mean myocardial ECV in LGE (-) segments in Group A+B was compared to that of controls. The mean myocardial ECV in Group A was compared to that of LGE (-) segments in Group B. The correlation between LV systolic function and the mean myocardial ECV of the whole myocardium was evaluated in all groups.

\section{Results}

Among the 41 DCM patients, 22 were in Group A, and 19 were in Group B. The mean ECV of DCM patents ( $\mathrm{n}=$ 41, 568 segments, $30.7 \% \pm 5.9$ ) was significantly higher ( $\mathrm{p}$ $<0.001)$ than that of the control group $(\mathrm{n}=10,157$ segments, $25.6 \% \pm 3.2$ ). The ECV was inversely related to LVEF in Group A $(r=-0.551, p=0.008)$, Group B $(r=$ -0.525, $\mathrm{p}=0.021)$, and Group $\mathrm{A}+\mathrm{B}(\mathrm{r}=-0.550, \mathrm{p}<0.001)$.

\section{Conclusions}

The ECV measured by MRI could be a useful parameter in evaluating diffuse myocardial changes in DCM patients.

\section{Authors' details}

'Department of Radiology, Research Institute of Radiological Science, Severance Hospital, Yonsei University College of Medicine, Seoul, Korea (the Republic of. ${ }^{2}$ Department of Radiology, Research Institute of Radiological Science, Gangnam severance Hospital, Yonsei University College of Medicine, Seoul, Korea (the Republic of.

Published: 3 February 2015

doi:10.1186/1532-429X-17-S1-P288

Cite this article as: Im et al: Extracellular volume fraction in dilated cardiomyopathy patients without obvious late gadolinium enhancement: comparison with healthy control subjects. Journal of Cardiovascular Magnetic Resonance 2015 17(Suppl 1):P288. 